\title{
Calibration methodology of a vision system for measuring the displacements of long-deck suspension bridges
}

\author{
Carlos Almeida Santos_,_,y, Carlos Oliveira Costa1 and Jorge Pereira Batista2 \\ 1Laborato'rio Nacional de Engenharia Civil, Lisboa, Portugal \\ 2Faculdade de Cie^ncias e Tecnologia da Universidade de Coimbra, Coimbra, Portugal
}

\section{SUMMARY}

Structural health monitoring is an emergent powerful diagnostic tool that can be used to identify and to prevent possible failures of the various components that comprise an infrastructure. In a suspension bridge,

the measurement of the vertical and transversal displacements plays an important role for its safety evaluation. Considering the restrictions usually found on these structures, an enhanced solution comprises a non-contact vision-based measurement system with dynamic response, accuracy and amplitude range well suited to the physical phenomenon to measure.

A methodology to perform the vision system calibration is described, which can be carried out in situ, while the deck is moving, requiring little effort and a minimum set of information. The key idea is to use the factorization method to get an initial estimation of the object shape and camera's parameters, and to incorporate the knowledge about the distances between the calibration targets in a non-linear optimization process to achieve a metric shape reconstruction and optimize the camera's parameters estimation. Results obtained by numerical simulation experiments are reported, showing the negligible influence of noisy calibration data in the calibration performance and also the robustness of the calibration process on the camera's parameters estimation. Tests carried out to assess the calibration and tracking of the bridge deck motion showed that, even in an environmental severely affected by noise, it is possible to measure the vertical and transversal displacements of the bridge deck with excellent levels of accuracy.

Copyright 2011 John Wiley \& Sons, Ltd.

Received 15 December 2009; Revised 27 October 2010; Accepted 20 November 2010

KEY WORDS: vision metrology; suspension bridge; non-contact measuring system; long-range photogrammetry; structural health monitoring; displacements; affine 